\title{
Autonomous Learning for Efficient Resource Utilization of Dynamic VM Migration
}

\author{
Hyung Won Choi ${ }^{1}$, Hukeun Kwak ${ }^{2}$, Andrew Sohn ${ }^{1}$, Kyusik Chung ${ }^{2}$ \\ ${ }^{1}$ Computer Science Department \\ New Jersey Institute of Technology \\ Newark, NJ 07102, U.S.A \\ hyung.choi@njit.edu, sohn@cs.njit.edu \\ ${ }^{2}$ School of Electronic Engineering \\ Soongsil University \\ 511 Sangdo-dong, Dongjak-gu, Seoul, Korea \\ \{gobarian, kchung\}@q.ssu.ac.kr
}

\begin{abstract}
Dynamic migration of virtual machines on a cluster of physical machines is designed to maximize resource utilization by balancing loads across the cluster. When the utilization of a physical machine is beyond a fixed threshold, the machine is deemed overloaded. A virtual machine is then selected within the overloaded physical machine for migration to a lightly loaded physical machine. Key to such threshold-based VM migration is to determine when to move which VM to what physical machine, since wrong or inadequate decisions can cause unnecessary migrations that would adversely affect the overall performance. We present in this paper a learning framework that autonomously finds and adjusts thresholds at runtime for different computing requirements. Central to our approach is the previous history of migrations and their effects before and after each migration in terms of standard deviation of utilization. We set up an experimental environment that consists of extensive real world benchmarking problems and a cluster of 16 physical machines each of which has on average eight virtual machines. We demonstrate through experimental results that our approach autonomously finds thresholds close to the optimal ones for different computing scenarios and that such varying thresholds yield an optimal number of VM migrations for maximizing resource utilization.
\end{abstract}

\section{Categories and Subject Descriptors}

C.5.5 [Computer System Implementation]: Servers; D.4.8 [Operating Systems]: Performance - measurements.

\section{General Terms}

Measurement, Performance, Experimentation.

\section{Keywords}

Virtualization, Virtual machines, OS migration, Linux cluster, Resource utilization.

Permission to make digital or hard copies of all or part of this work for personal or classroom use is granted without fee provided that copies are not made or distributed for profit or commercial advantage and that copies bear this notice and the full citation on the first page. To copy otherwise, or republish, to post on servers or to redistribute to lists, requires prior specific permission and/or a fee.

ICS'08, June 7-12, 2008, Island of Kos, Aegean Sea, Greece.

Copyright 2008 ACM 978-1-60558-158-3/08/06..\$5.00.

\section{INTRODUCTION}

The computational demands of an organization change over time for various reasons. The ability to offer required computing resources under unpredictable growth demands is challenging with the results often being unreliable. A good example is preparing for Monday morning surges. As people start working, there is enormous stress on a multitude of servers and applications. By mid morning the peak will have come and gone. How high the level reaches is unpredictable to some extent. Typically web servers, mail servers, file servers, and database servers are the most heavily taxed systems during these upswings in traffic [1]. To prepare for such a spike in usage, enterprises and organizations are often forced to greatly over-provision computational infrastructure, even though the average utilization of resources maybe typically below $15 \%-20 \%$ [25].

Research and development efforts conducted to address this utilization problem have led to adaptive infra-structure using resource virtualization [25]. Virtualization techniques have a long history [10] of research, development and implementation, dating back to the IBM System/360 and 370 [7]. Commercial products include VMware [23], Microsoft's Virtual Server [14], and Sun Containers/zones [22]. VMware, a part of EMC, is a virtual machine software suite allowing users to create and execute virtual computers not only on the host OS but more importantly on bare-metal x86 hardware [23]. MS Virtual Server is the virtual machine solution targeted specifically to Windows environment [14]. As built-in virtualization technology in Solaris 10, Sun containers share the same OS images/drivers running on top of the Solaris kernel to provide multiple private-execution environments [22].

Virtualization projects pursued in the open-source community include Xen [2,28], User-mode Linux (UML) [8,9], Bochs [13], Plex86 [16], and others [20]. Xen, apart from XenSource which is now part of Citrix, is a software layer, called para-virtualization, which enables guest operating systems to run on a physical machine [2]. User-Mode Linux (UML) is an application software layer that enables guest Linux operating systems to run on the host Linux operating system. UML, being an application from the host's perspective, requires no modification of the underlying host kernel [8].

When many virtual machines are deployed on a cluster of machines, controlling them can often cause more problems than solutions [18]. Some machines will have many overloaded virtual machines while others do not. To solve its imbalance problem, virtual machine migration methods have been introduced, including Xen Live migration [6], VMware's VMotion [24], 


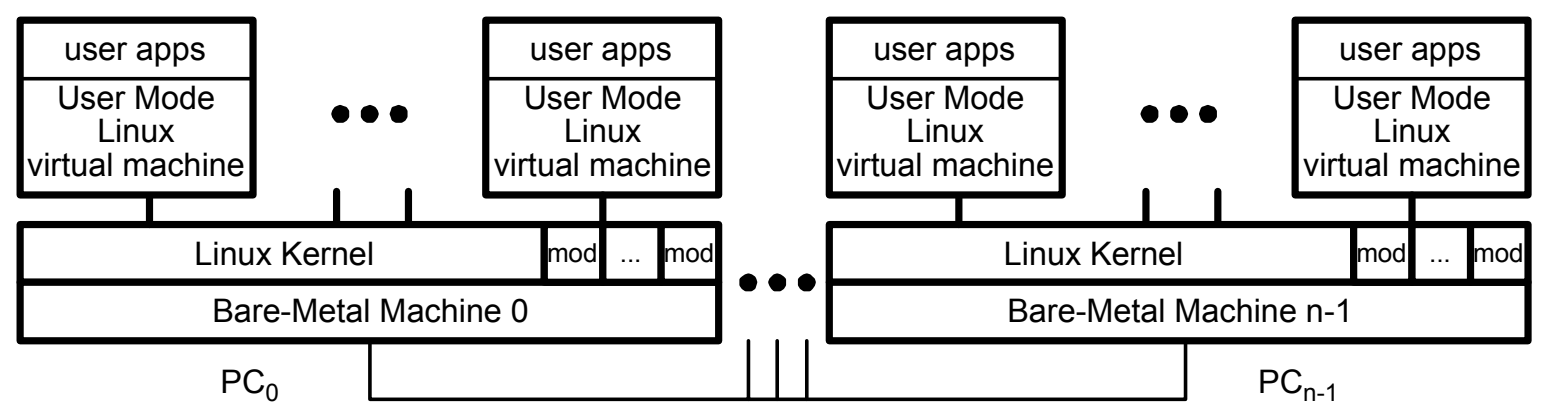

Figure 1. Overview of the system.

Checkpointing [4] such as Berkeley Lab Checkpointing and Restart [12], etc. Studies based on Xen's Live migration explicate the benefits of using runtime VM migration. In particular, the Proactive Fault Tolerance method reported in [15] demonstrates the difference between Xen's Live migration and Stop\&Copybased migration using NAS Parallel Benchmark while Sandpiper [27] illustrates how hot spots can be mitigated using Xen's Live migration in a datacenter environment with high web traffic.

When the utilization of a physical machine is beyond a fixed threshold, the machine is deemed overloaded. A virtual machine within the physical machine will be selected to move to a lightly loaded physical machine. This fixed threshold can become critical to overall performance of the cluster. Not only does finding such thresholds require much time and administrator's continuous intervention but more importantly the resulting threshold may not work for different situations since the computing demands change over time.

It is precisely the purpose of this paper to introduce a framework that autonomously finds and adjusts thresholds at runtime with no human intervention. Central to our approach is the history of migrations along with its impact on performance before and after each migration. For each migration the standard deviation of resource utilization of the cluster is recorded before and after migration. This recording and learning provides a reference point for future decisions. After each learning, the thresholds incrementally approach to the optimal ones.

The paper is organized as follows. Section 2 presents our approach to dynamic migration of virtual machines. Section 3 explains our experimental environment including benchmarking suites, workload definition, benchmarking methodology, and workload distribution. Section 4 lists experimental results and discusses the results. Section 5 presents performance analysis and the last section concludes our work.

\section{DYNAMIC MIGRATION FRAMEWORK 2.1 Overall}

The framework shown in Figure 1 consists of a cluster of Linux PCs connected through a network. Each bare-metal machine is comprised of a Linux operating system and virtual machines with our own modules. Vital statistics such as CPU usage are monitored and collected in a predetermined interval by the kernellevel modules. Running on top of the Linux kernel (or host operating system) are multiple User Mode Linux Virtual Machines (UML VM or guest operating systems). The number of UML VMs a machine can accommodate depends on the amount of resources in each machine, in particular memory, CPU speed, and disk. User applications run on UML VMs. A user-level application running on our own kernel modules determines how the currently active VMs are autonomously migrated.

The detailed description of UML VMs and kernel-level modules is illustrated in Figure 2. Each PC consists of VMs, SBUML interface and two to three modules depending on its role. ScrapBook UML (SBUML) [17,19], an extension to UML, provides an OS checkpointing capability that is essential to our dynamic migration framework. With modifications, we seamlessly integrate SBUML into our dynamic migration framework. A PC is designated as the master PC for making decisions on migration while the rest as workers. $\mathrm{PC} 0$, the master $\mathrm{PC}$, has three modules of monitoring, learning, and migration

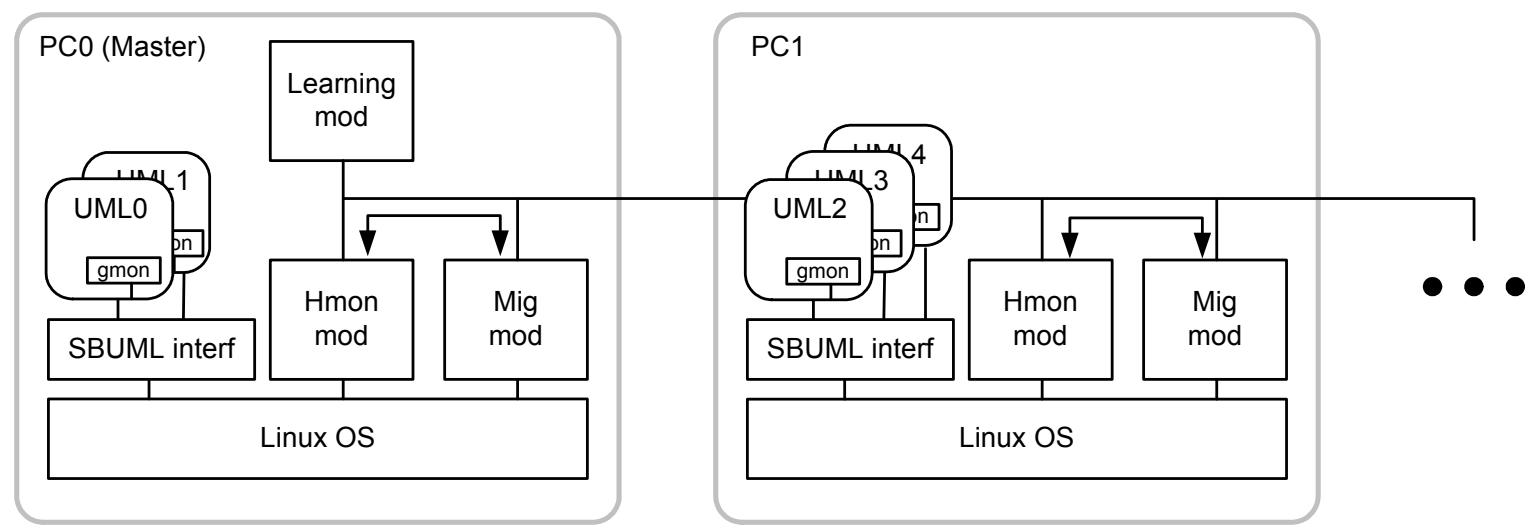

Figure 2. Logical organization. Hmon=host monitor, gmon=guest $\operatorname{monitor,~} \bmod =$ module, interf $=$ interface. 
while the remaining worker PCs each have two modules, monitoring and migration. Included in each UML VM is a monitoring module for the VM's utilization statistics, not the host's utilization. SBUML interface provides a bridge between the host machine and the guest VMs for checkpointing. Monitoring modules periodically collect utilization statistics for both host and guest machines. The learning module of the master PC checks if any machines are overloaded. If any machine is indeed overloaded, a VM is selected for migration. Migration modules actually move the selected VM to the target PC.

\subsection{Monitoring modules}

Monitoring modules periodically query OS kernel for CPU and memory usage. Two types of monitoring modules are used: host monitor hmon and guest monitor gmon. Hmon runs on each host OS to collect the host's usage while gmon on each and every guest OS (VM) to collect guest VM's utilization. Gmon collects statistics from the guest's perspective which is different from the host's.

Host CPU utilization is computed using the "/proc/stat" file of the host OS. Every second the difference is computed and accumulated for a fixed period of time.

At the end of this period, the statistics are averaged and sent to the learning module. Computing guest OS utilization is similar to host utilization. The guest OS utilization is recorded on the log file of the host OS's file system. Writing this allows the host OS to access the guest VM's statistics.

\subsection{Learning module}

The Learning module determines migration using history matrix along with other information. Each entry of the history matrix consists of the following: standard deviation of CPU utilization before migration, host CPU Utilization, Source Host, Destination Host, and standard deviation of CPU utilization after migration. The learning module works as follows (Figure 3):

Step 1: Obtain CPU utilization for all the hosts and VMs. Find the standard deviation of the host CPU utilization.

Step 2: Check if there is an entry in the matrix that is the same as the current situation in terms of both the current standard deviation and host CPU utilization. If it does exist, check if the standard deviation after migration is less than the current one. If true, it is projected that migration would help. An actual migration will ensue in Step 4. If there is no such entry in the matrix or the standard deviation after migration is higher, Step 3 will be performed.

Step 3: All the possible migrations are simulated to find the estimated standard deviations after migration. Check if the smallest standard deviation is smaller than the current one. If true, a migration will ensue in Step 4. Otherwise, go back to Step 1 to pick another candidate host machine for migration.

Step 4: An actual migration takes place using the Source Host and the Destination Host determined in Step 2 or 3. A VM with the lowest standard deviation after migration is selected from the host machine for migration. After actual migration, an entry in the history matrix will be added or updated with the current information (standard deviation of CPU utilization before migration, host CPU Utilization, Source Host, Destination Host, and standard deviation of CPU utilization after migration).

\subsection{Migration modules}

Migration modules move virtual machines. Upon receiving the decision results from the master $\mathrm{PC}$, the migration module of the source PC initiates the first half of migration by creating a snapshot of the selected virtual machine and packing the snapshot, followed by sending to the destination PC. The migration module at the target $\mathrm{PC}$ unpacks the snapshot received and resumes the execution of the guest OS.

\section{EXPERIMENTAL ENVIRONMENT}

\subsection{Overview}

To test the proposed approach we have set up a cluster of 16 PCs connected through a $100 \mathrm{Mbps}$ switch. Each PC has Pentium 4 $2.26 \mathrm{GHz}$ with 1 to $2 \mathrm{~GB}$ memory. Linux kernel 2.4.20-6 version is used as the host operating system. Each PC can have up to 16 UML virtual machines each of which uses Linux kernel 2.4.23. The total number of VMs ranges 68 to 136 depending on the configuration. All VMs each have $128 \mathrm{MB}$ of memory allocated. The kernel for VMs consists of 2.4.23 Linux kernel, UML patch, and SBUML patch. SBUML is used for freezing and restoring VMs at runtime. The size of virtual machine file system is typically approximately $100 \mathrm{MB}$. Table 1 summarizes the key parameters used in the migration framework.

\subsection{Benchmark suites}

Two benchmark suites of MiBench and SPLASH-2 are used to

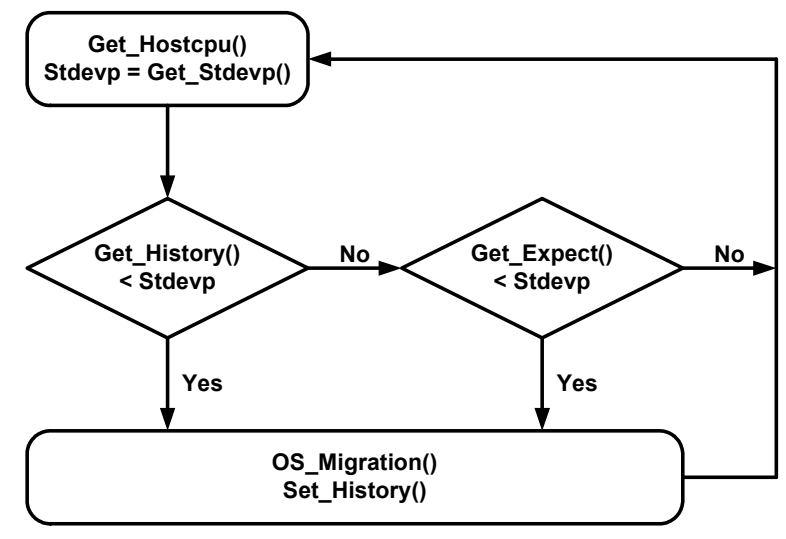

Figure 3. Learning procedure. 
Table 1. Key parameters for the framework.

\begin{tabular}{|l|l|l|}
\hline Migration thresholds (cpu utilization \%) & $55 \%, 65 \%, 75 \%, 85 \%$ \\
\hline \multirow{2}{*}{$\begin{array}{l}\text { Learning } \\
\text { parameters }\end{array}$} & CPU standard deviation types & 11 \\
\cline { 2 - 3 } & History matrix size & $1100=11 \times 100$ \\
\hline \multirow{2}{*}{ Hosts } & Monitor collected cpu util \& calculated & every second from /proc/stat on hosts \\
\cline { 2 - 3 } & Monitoring period & 20 seconds \\
\hline \multirow{2}{*}{ Guests } & Guest Monitor collect & every second from /proc/stat on guests \\
\cline { 2 - 3 } & Guest Monitor collecting duration & 3 seconds \\
\hline Number of guests per host & 4,8 \\
\hline Number of guests on the overloaded host & 8,16 \\
\hline \multicolumn{2}{|l|}{ Total number of guests } & 68,136 \\
\hline
\end{tabular}

test the proposed framework. We selected 30 applications from these suites, all of which run on virtual machines. MiBench [11] is a widely used embedded benchmark suite, which consists of 27 applications. We selected the following 21 applications from MiBench to evaluate the framework: Automotive Class (basicmath, bitcount, qsort, susan), Consumer Class (jpeg, lame, mad, tiff2bw), Network Class (dijkstra, patricia), Office Class (ispell, rsynth, stringsearch), Security Class (blowfish, pgp, rijndael, sha), and Telecomm Class (adpcm, CRC32, FFT, gsm). Each of the above applications requires various input data and parameter settings. It is not practical to list the benchmarking details, except to indicate that we have expended significant time and efforts to develop a set of benchmarking scenarios to closely simulate a realistic computing environment. The second benchmark suite we used is SPLASH-2 (Stanford Parallel Applications for Shared Memory) [26]. The following nine applications are used in our experimentations: ocean contiguous and non-contiguous, cholesky, fft, lu contiguous and noncontiguous, radix, water-nsquared, and water-spatial.

Our benchmarking consists of finding three types of workloads: unit, group, and total. Unit workload is defined as the configuration of an application that takes a predefined wallclock execution time within $1-2 \%$ tolerance. Group workload is comprised of 100 unit workloads, assembled from various different applications but not necessarily from all 30 applications. Total workload is defined as the number of groups. For our experiments it is set to 10 .

\subsection{Initial Workload Distribution}

The total workload defined above is divided and assigned to each of the 16 PCs. Table 2 lists eight scenarios of how the total workload is assigned to the cluster. In particular, the table shows five different ways of overloading one PC, PC1. The first row indicates that $\mathrm{PC} 1$ executes $40 \%$ of the total workload while the remaining $15 \mathrm{PCs}$ each execute $4 \%$. The last row, Scenario 5 , is an extreme case where PC1 is now assigned $80 \%$ of the total workload while all the other PCs each have merely $1.33 \%$ assigned. Again the table shows how one PC is overloaded.

Recall that each PC can have multiple VMs. The workload assigned to each PC is equally assigned to the virtual machines within the PC. This assignment is done in terms of workload units. Now that each and every PC and VM have its work assigned, the cluster starts executing the applications according to the initial distribution. As the execution progresses, the utilization of each PC will change, some will be highly overloaded while some under utilized. VM migrations will ensue to balance the loads across the cluster.

Table 2. Initial workloads assignment for overloading 1 PC.

\begin{tabular}{|c|c|c|c|c|c|c|}
\hline Scenari & $\%$ & PC0 & PC1 & PC2 & $\ldots$ & PC15 \\
\hline 1 & $40 \%$ & 4 & 40 & 4 & $\ldots$ & 4 \\
\hline 2 & $50 \%$ & 3.33 & 50 & 3.33 & $\ldots$ & 3.33 \\
\hline$\ldots$ & $\ldots$ & $\cdots$ & $\cdots$ & $\cdots$ & $\cdots$ & $\cdots$ \\
\hline 5 & $80 \%$ & 1.33 & 80 & 1.33 & $\ldots$ & 1.33 \\
\hline
\end{tabular}

\section{EXPERIMENTAL RESULTS}

\subsection{No migration vs. migration}

We present experimental results in terms of three key parameters: (a) the percentage of workload each PC is initially assigned, (b) the average number of VMs on each PC, and (c) the threshold for migration ranging $55 \%$ to $85 \%$. In Table 2 , we had only one PC overloaded with $40 \%$ of the total amount of work while the rest with $60 \%$ (Scenario 1). We now have that particular PC overloaded with $w \%$ of the total workload, where $w$ varies $40 \%$ to $80 \%$. $100 \%$ work indicates that the overloaded PC is assigned $100 \%$ of the total work while others do nothing. 
Table 3. Sample completion times in seconds for the total workload under various different scenarios. mig=migration. Initially, $60 \%$ of the total workload is assigned to the overloaded PC1.

\begin{tabular}{|c|c|c|c|c|c|c|c|c|c|c|c|c|c|c|c|c|c|}
\hline \multicolumn{2}{|c|}{ PC number } & 0 & 1 & 2 & 3 & 4 & 5 & 6 & 7 & 8 & 9 & 10 & 11 & 12 & 13 & 14 & 15 \\
\hline \multirow{5}{*}{$\begin{array}{l}4 \mathrm{VMs} \\
\text { per PC }\end{array}$} & No mig & 408 & 473 & 409 & 415 & 416 & 412 & 418 & 426 & 426 & 430 & 433 & 432 & 460 & 427 & 42 & 433 \\
\hline & Mig (th55) & 102 & 129 & 123 & 102 & 421 & 120 & 438 & 425 & 426 & 466 & 433 & 433 & 459 & 426 & 42 & 949 \\
\hline & Mig (th65) & 418 & 108 & 930 & 444 & 120 & 416 & 418 & 116 & 432 & 112 & 436 & 126 & 461 & 433 & 96 & 436 \\
\hline & Mig (th75) & 418 & 105 & 120 & 425 & 422 & 442 & 115 & 503 & 431 & 110 & 438 & 949 & 461 & 904 & 44 & 116 \\
\hline & Mig (th85) & 980 & 105 & 421 & 420 & 421 & 421 & 111 & 119 & 915 & 113 & 432 & 431 & 460 & 429 & 42 & 107 \\
\hline \multirow{5}{*}{$\begin{array}{l}8 \mathrm{VMs} \\
\text { per PC }\end{array}$} & No mig & 147 & 380 & 150 & 154 & 156 & 165 & 172 & 175 & 177 & 179 & 185 & 188 & 215 & 189 & 19 & 320 \\
\hline & Mig (th55) & 973 & 907 & 924 & 114 & 116 & 608 & 106 & 867 & 133 & 831 & 119 & 901 & 210 & 129 & 54 & 102 \\
\hline & Mig (th65) & 145 & 924 & 104 & 563 & 131 & 810 & 932 & 537 & 123 & 130 & 864 & 114 & 134 & 609 & 19 & 118 \\
\hline & Mig (th75) & 982 & 136 & 107 & 129 & 619 & 714 & 122 & 539 & 128 & 938 & 787 & 745 & 935 & 862 & 19 & 135 \\
\hline & Mig (th85) & 117 & 136 & 576 & 931 & 103 & 161 & 125 & 132 & 171 & 533 & 121 & 876 & 203 & 661 & 19 & 119 \\
\hline
\end{tabular}

Table 3 lists a small portion of the completion times with migration and no migration and for the total workload defined earlier using various different parameter settings. The first row indicates actual PC numbers, not the number of PCs. The next row labeled "4 VMs per PC" lists completion times with migration on (with thresholds) and off for $60 \%$ of the total workload initially assigned to $1 \mathrm{PC}$ with four VMs per PC. The third row labeled "8 VMs per PC" lists completion times with eight VMs per PC.

Figure 4 plots the completion times of Table 3 for the $60 \%$ initial overload with various thresholds. The $\mathrm{x}$-axis is $\mathrm{PC}$ numbers while the y-axis is the completion time in seconds. The thin (cyan) lines indicate the results with no migration while the other lines with migration using various thresholds. The completion times without migration are not consistent as expected since the overloaded machines will take longer to complete the workload while under utilized machines will finish quickly and remain idle. On the other hand, the completion times are consistent across all the machines when migration is turned on.

Consider Figure 4(a), where each PC has four VMs on average. Note that PC1 is overloaded with $60 \%$ of the total workload in the beginning. As expected, PC1 took close to 5,000 seconds to complete the workload while all 15 other machines each took only about 400 seconds, leaving $\mathrm{PC} 1$ being the critical path. When migration is turned on, however, all the 16 machines exhibit relatively consistent completion times with no apparent aberrations or critical path since the $\mathrm{VM}(\mathrm{s})$ on the overloaded machine were migrated to under utilized machines. The small peaks and valleys are due to the resolution, i.e., the average number of virtual machines in each PC.

When the average number of VMs per PC is increased to eight in Figure 4(b), we obtain results consistent with Figure 4(a) with a few exceptions. As expected, PC1 is still the critical path, taking 4000 seconds to complete while all 15 other machines each took only about 300 seconds. Earlier in Figure 4(a), we found that each of the 15 PCs took approximately 400 seconds. The difference of 100 seconds is due to the fact that a larger number of VMs allows a larger amount of overlapping between computing and communication. In other words, finer granularity of VMs increases an opportunity for overlapping in packing, sending, receiving and resuming among VMs.

We also observe find from the two plots that the fluctuation for 8$\mathrm{VM}$ is smaller than that for $4-\mathrm{VM}$, except when the threshold is $85 \%$. This high threshold of $85 \%$ strongly discourages migrations, resulting in higher discrepancy between those overloaded and under-loaded. We will see later that this high threshold ultimately limits the efficiency of resource utilization.

We have seen in Figure 4 the impact of migration on completion times when $60 \%$ of the total workload is initially assigned to one
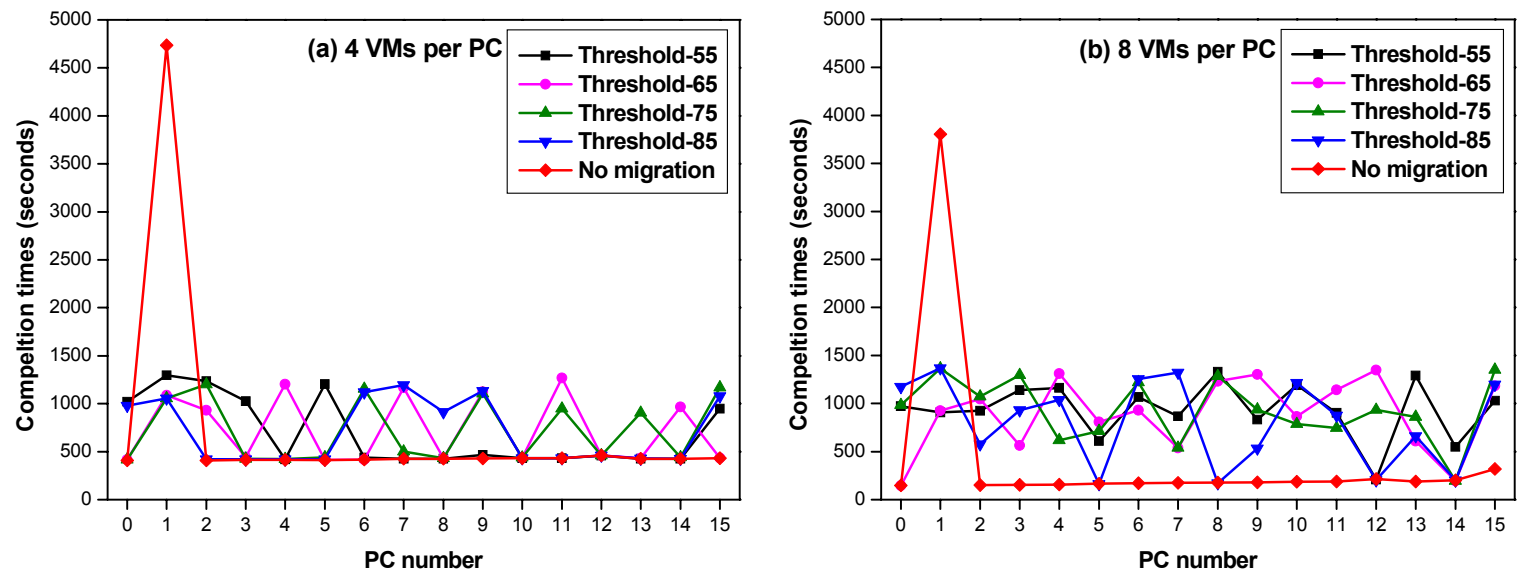

Figure 4. Comparison of completion times for $60 \%$ of the total workload initially assigned to one PC with migration on and off: (a) $4 \mathrm{VMs}$, (b) $8 \mathrm{VMs}$ per PC. 

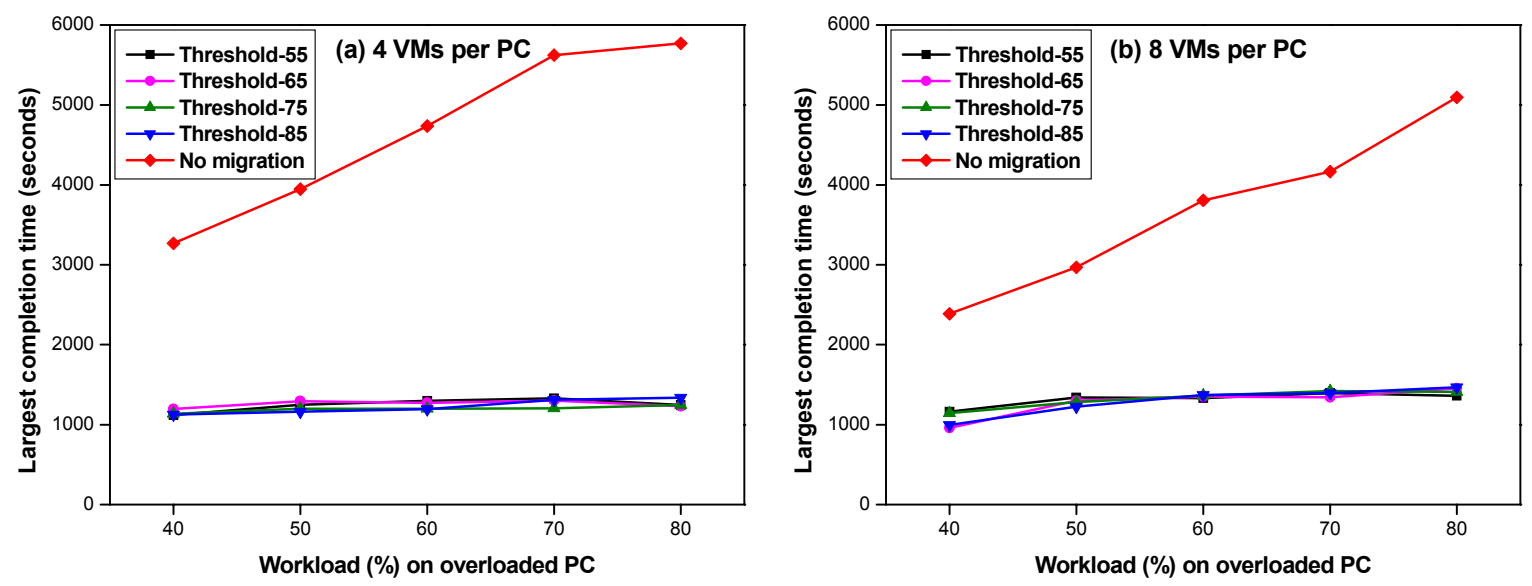

Figure 5. Overall completion times: (a) 4 VMs, (b) 8 VMs per PC

PC. Figure 5 shows the impact with initial workload ranging $40 \%$ to $80 \%$. Again Figure 5(a) is for one PC initially overloaded and each $\mathrm{PC}$ with four virtual machines on average while Figure 5(b) is for each PC with eight virtual machines. The $\mathrm{x}$-axis is the percentage of total workload initially assigned to the overloaded PC while the y-axis is the largest completion time. We have plotted in Figure 4 that some PCs finish early while some finish late depending on the machine's load. However, the completion time in Figure 5 is the critical path of a PC that took the longest time. The plot has five curves. The top curves are completion times with no migration while the bottom ones are with migration using the thresholds of $55,65,75$, and $85 \%$.

The plots clearly indicate that the results with migration are far superior to that with no migration. With no migration we find that the longest completion time by the initially overloaded PC increases as the workload of the overloaded PC increases. When migration is turned on, however, the workloads of the initially overloaded PC have little impact on the overall completion time. In fact the overall completion times with migration appear close to each other because the no-migration results are very large. In what follows, we present the differences.

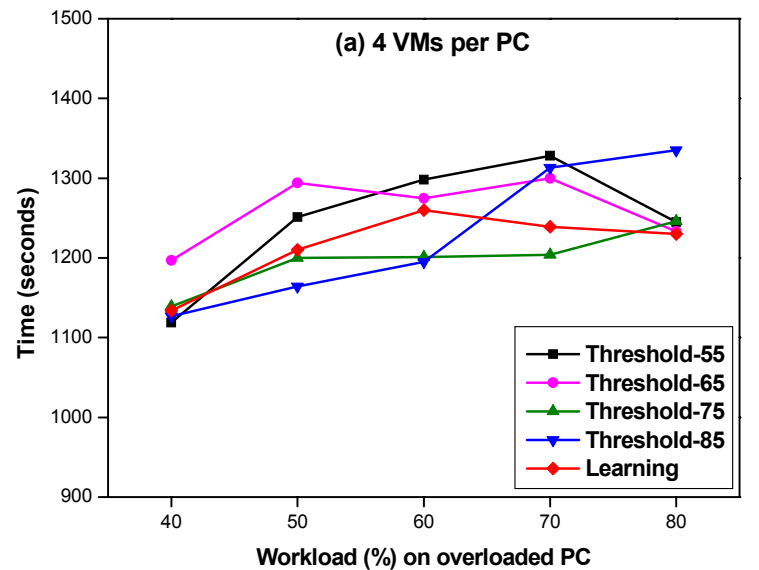

\subsection{Thresholds vs. learning}

Figure 6 shows the differences between thresholds and learning. Figure 6(a) is for $4 \mathrm{VMs}$ and (b) for $8 \mathrm{VMs}$ per PC on average. To be more precise, the overloaded PC in Figure 6(a) has eight VMs while the remaining $15 \mathrm{PCs}$ each have four VMs, resulting in $8+$ $4 \times 15=68 \mathrm{VM}$ on the cluster. The $x$-axis shows the percentage of the total workload on the initially overloaded PC while the $y$-axis shows execution time in seconds.

The figure shows five curves: four fixed thresholds and learning. It is clear from the figure that varying the threshold results in mixed execution times. Consider when the overloaded PC is initially set to $40 \%$ of the total workload. The learning approach and the thresholds of 55, 75 and 85 show similar execution times (approximately 1120 second) while the threshold of 65 performs poorly (close to 1200 seconds). However, this observation does not hold for different initial workloads. When the overloaded PC is initially set to $80 \%$ of the total workload, the threshold of 85 shows the worst performance (over 1300 seconds) while the other three shows almost the same execution time.

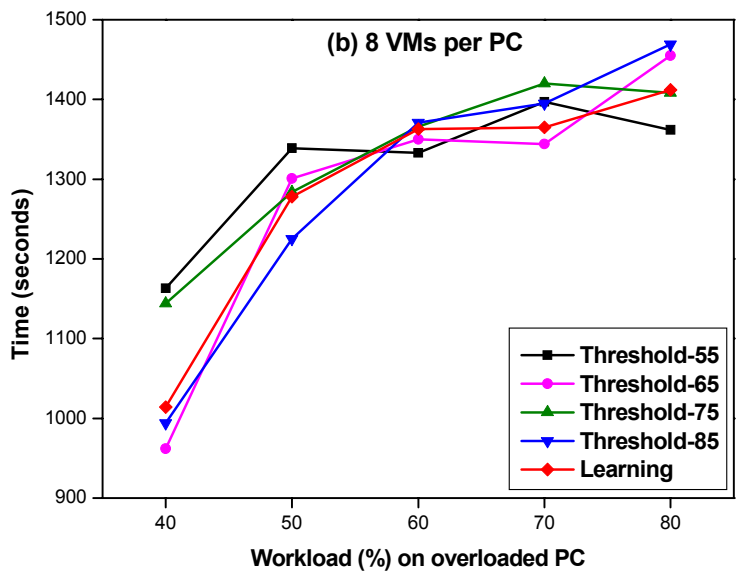

Figure 6. Execution time in seconds: (a) 4 VMs, (b) 8 VMs on each PC 


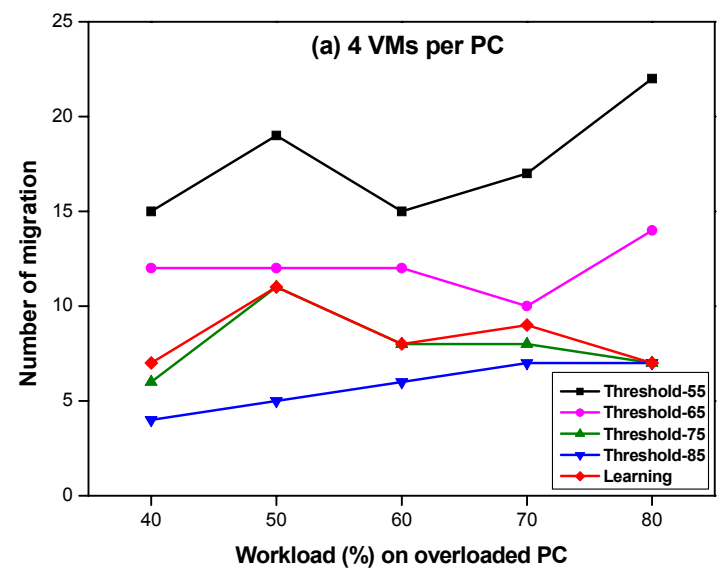

Figure 7. Number of migrations: (a) 4 VMs, (b) 8 VMs on each PC.

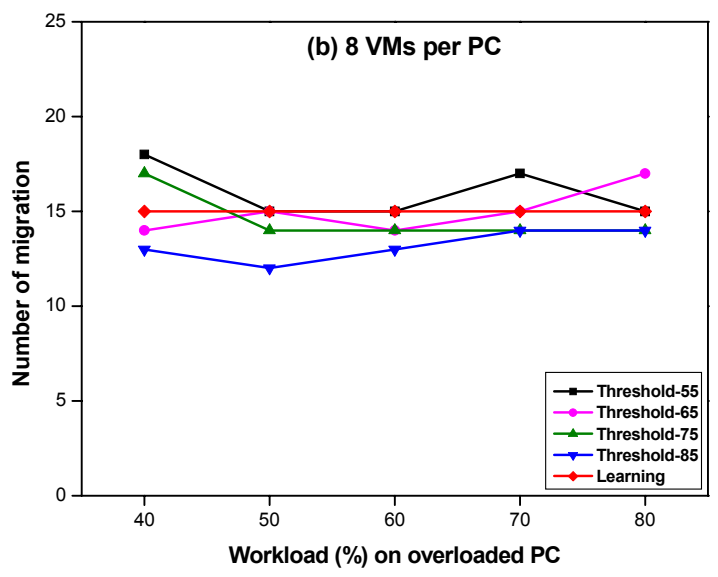

learning yields a moderate number of migrations regardless of the computing scenarios.

Consider the results for Threshold-55 and Threshold- 85 in Figure 7(a). The number of migrations for Threshold-55 is three to four times higher than that for Threshold-85. The main reason for this large difference is "easy" migration. Since the threshold is low, migration takes place often and unnecessarily, resulting in higher overhead. On the other hand, the high threshold of $85 \%$ severely limits migrations while reducing migration overhead. However, the savings in overhead does not compensate the severe imbalance between physical machines, resulting in lower performance. Learning solves the dilemma. The results show that learning yields a moderate number of migrations by finding right thresholds for different computing scenarios. This is an indication that the learning approach utilizes resources effectively and efficiently.

The number of VMs affected the number of migrations. When the number of VMs was doubled, the change in the number of migrations has been substantially reduced, as illustrated in Figure 7. For 4-VMs shown in (a), the number of migrations varies substantially. However, for 8 -VMs, the variation is small. The difference in variations is in the fact that the smaller number of VMs causes the Ping-Pong problem. When a VM is moved, the receiving $\mathrm{PC}$ can be more susceptible to overloading than other PCs, in which case the VM that was just moved may be moved again either back to the original source PC or yet another. This ping-pong behavior thus results in a higher number of migrations. For 8-VMs however, this ping-pong effect is not pronounced since the granularity is smaller and a VM can fit into the receiving PC.

\subsection{CPU utilization}

CPU utilization indicates how loads are distributed across the cluster. Figure 8 shows CPU utilization for individual PCs. The $x$ axis is PC numbers while the $y$-axis is average CPU utilization for the duration of each experiment, where each experiment typically takes tens of minutes to over an hour (See Table 3 for sample completion times). 

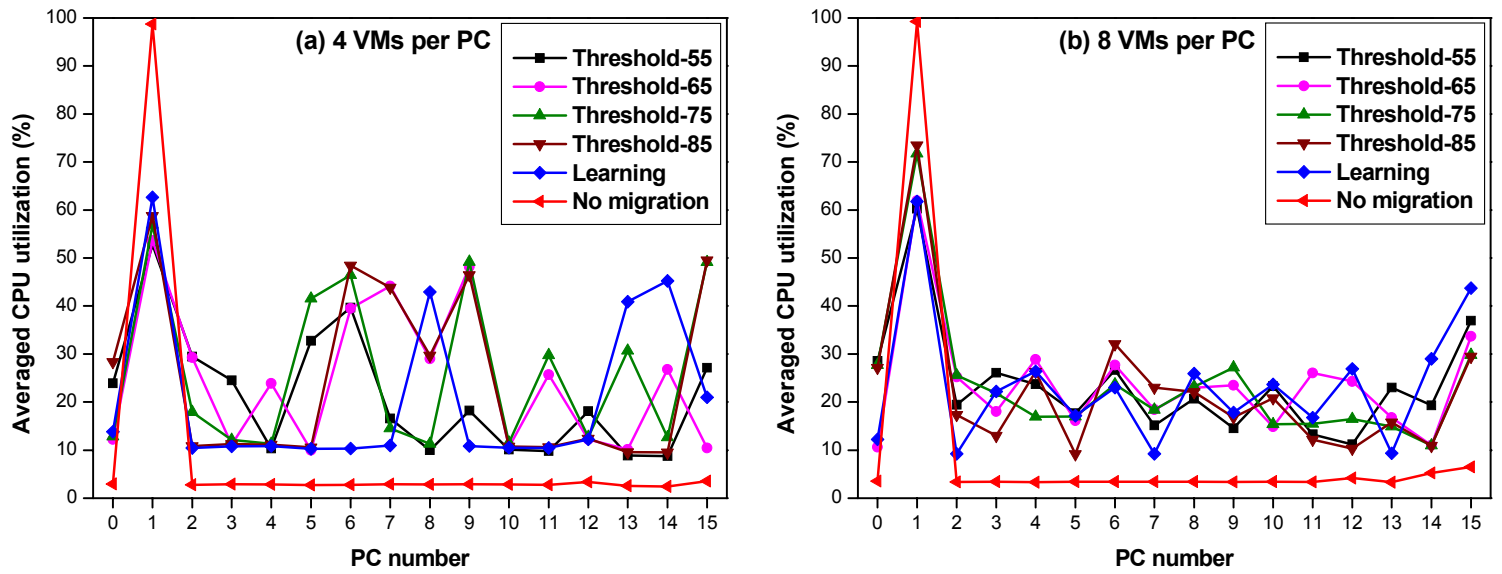

Figure 8. Sample CPU utilization for the $60 \%$ workload initially assigned to PC1: (a) 4 VMs, (b) 8 VMs on each PC.

As expected, no migration shows poor CPU utilization. PC1 which is initially assigned $60 \%$ of the total workload maintained $100 \%$ utilization throughout while other 15 PCs finished the work early and stayed idle, resulting in close to zero utilization. On the other hand, the thresholds and learning approaches show fairly consistent CPU utilization. Learning exhibits more consistent CPU utilization than other approaches for 4-VMs, except the three PCs (PCs 8, 13 and 14). This aberration is due in part to the fact that the number of VMs is small and there is little room to maneuver. When the number of VMs is doubled, this aberration no longer exists. In general, we find that doubling the number of VMs reduces the average utilization for the given amount of workload. This reduced average utilization further attests to the fact that increasing the number of VMs proportionally increases the efficiency of resource utilization, which will be discussed below.

\subsection{Resource utilization efficiency}

The results presented above have indicated that the learning method helps find the right thresholds at runtime to increase resource utilization. We wish to identify how efficient the learning approach is compared to the fixed threshold approach and no migration. We define the efficiency for resource utilization $E$ as follows:

$$
E=S_{4-V M, n o-m i g} / S,
$$

where Sum $S$ is the resource utilization required to complete the total workload and is defined as $S=\Sigma U_{\mathrm{i}} * T_{\mathrm{i}}$, where $U_{\mathrm{i}}$ is the total cpu utilization and $T_{\mathrm{i}}$ is the total time taken for $\mathrm{PC}_{\mathrm{i}}$.

Table 4 lists total resource utilization for individual PCs and Figure 9 plots efficiency. The last row of Table 4 sums all the resource usage for each approach. We find from the table that learning yields the lowest sum (8-VMs) or the second lowest sum (4-VMs) while other approaches vary. For 4-VMs, the difference

Table 4. Resource utilization. The total resource usage for each approach is shown in the last row.

\begin{tabular}{|c|c|c|c|c|c|c|c|c|c|c|c|c|}
\hline & \multicolumn{6}{|c|}{$4 \mathrm{VMs}$} & \multicolumn{6}{|c|}{$8 \mathrm{VMs}$} \\
\hline & Thr-55 & Thr-65 & Thr-75 & Thr-85 & Learn & No-mig & Thr-55 & Thr-65 & Thr-75 & Thr-85 & Learn & No-mig \\
\hline $\mathrm{PC} 0$ & 24405 & 5112 & 5355 & 27783 & 5767 & 1210 & 27740 & 1540 & 27241 & 31893 & 1801 & 517 \\
\hline $\mathrm{PC} 1$ & 68469 & 58339 & 60175 & 62061 & 71296 & 467443 & 54647 & 57233 & 97983 & 100635 & 58216 & 377669 \\
\hline PC2 & 36388 & 27240 & 21534 & 4555 & 4324 & 1144 & 17981 & 26447 & 27469 & 9976 & 1356 & 507 \\
\hline PC3 & 25172 & 4942 & 5160 & 4733 & 4545 & 1199 & 29754 & 10151 & 28283 & 12122 & 29615 & 526 \\
\hline PC4 & 4341 & 28775 & 4769 & 4694 & 4596 & 1178 & 27610 & 37904 & 10486 & 27325 & 25908 & 514 \\
\hline PC5 & 39446 & 4159 & 18370 & 4442 & 4324 & 1119 & 10762 & 13082 & 12124 & 1478 & 19242 & 567 \\
\hline PC6 & 17380 & 16503 & 53638 & 54193 & 4363 & 1156 & 28468 & 25816 & 28889 & 40273 & 29791 & 591 \\
\hline PC7 & 7047 & 51546 & 7294 & 52333 & 4713 & 1235 & 13118 & 9892 & 9896 & 30472 & 1619 & 601 \\
\hline PC8 & 4241 & 12537 & 4883 & 27148 & 54006 & 1213 & 27559 & 28333 & 29776 & 3777 & 35318 & 601 \\
\hline PC9 & 8501 & 54206 & 54355 & 52639 & 4705 & 1244 & 12091 & 30600 & 25514 & 8976 & 16885 & 607 \\
\hline PC10 & 4369 & 4630 & 5057 & 4644 & 4582 & 1231 & 27798 & 12839 & 12080 & 25284 & 27268 & 629 \\
\hline PC11 & 4245 & 32549 & 28233 & 4564 & 4568 & 1206 & 11974 & 29824 & 11525 & 10661 & 12149 & 633 \\
\hline PC12 & 8294 & 5629 & 5850 & 5709 & 5650 & 1557 & 2348 & 32821 & 15390 & 2091 & 6035 & 903 \\
\hline PC13 & 3771 & 4386 & 27753 & 4115 & 48852 & 1094 & 29683 & 10225 & 12835 & 10411 & 1738 & 620 \\
\hline PC14 & 3731 & 25879 & 5578 & 4052 & 51427 & 1048 & 10576 & 2140 & 2143 & 2169 & 32161 & 1039 \\
\hline PC15 & 25737 & 4547 & 57421 & 53443 & 24540 & 1535 & 38011 & 39867 & 40317 & 35300 & 7060 & 2070 \\
\hline Sum $S$ & 285535 & 340980 & 365422 & 371109 & 302257 & 485811 & 370120 & 368712 & 391950 & 352843 & 306160 & 388593 \\
\hline
\end{tabular}


between the lowest (285535) and learning (302257) is $0.5 \%$, which is small enough to be considered as noise. Again the main reason for this low total utilization is due to the fact that the loads are equally distributed across the cluster. Figure 9 summarizes our study: learning thresholds at runtime gives the highest or close to the highest efficiency for resource utilization.

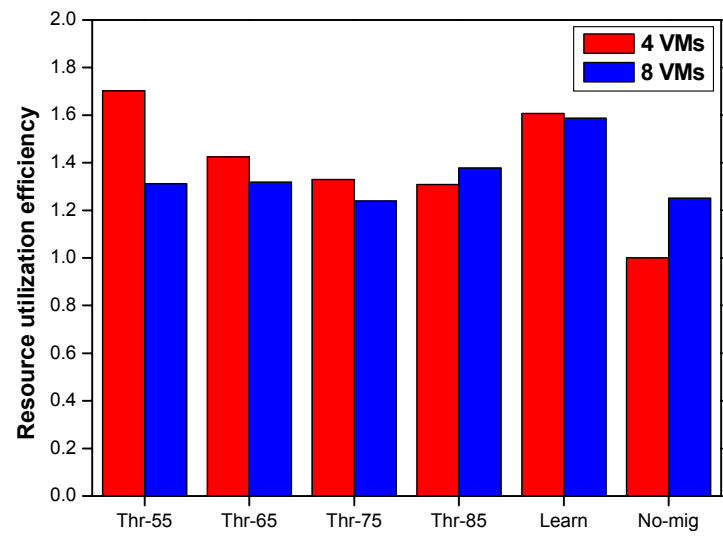

Figure 9. Resource utilization efficiency.

\section{CONCLUSIONS}

Dynamic migration of virtual machines is designed to maximize resource utilization by balancing loads across the cluster. In this paper, we have presented a learning framework that autonomously finds and adjusts the thresholds for determining candidate VMs for migration. We have set up an experimental environment with a cluster of 16 PCs. Each PC has on average 4 to 8 User Mode Linux (UML) VMs, resulting in the total of 68 to $136 \mathrm{VMs}$ depending on experimental settings. Each VM ran two suites of 30 benchmark programs that include applications for daily computing. Key to our approach is the learning module that autonomously finds and adjusts the thresholds for selecting source VMs and destination PCs using migration history and standard deviation of the CPU utilization of VMs.

We have performed numerous experimentations with three key parameters. First, the percentage of workload each PC is initially assigned varied from $40 \%$ to $80 \%$ of the total workload. Second, the average number of VMs on each PC varied four to eight. Third, the threshold for migration varied $55 \%$ to $85 \%$. Experimental results have demonstrated that (a) the overall performance with migration was far superior to that with no migration; (b) the learning approach consistently performed better than the threshold approach. We have found that learning yields moderate but consistent number of migrations by finding right thresholds for different computing scenarios. On the other hand, the fixed-threshold approach has yielded varying number of migrations for different computing scenarios, resulting in overall performance degradation; (c) the learning approach has yielded the highest or second highest efficiency in resource utilization among all the experimentations. In summary, we find that learning thresholds at runtime has indeed helped maximize the limited resources.

\section{REFERENCES}

[1] V. Almeida, R. Riedi, D. Menasce, W. Meira, F. Ribeiro, R. Fonseca, Characterizing and modeling robot workload on ebusiness sites, in Proc. ACM SIG METRICS, June 2001.

[2] P. Barham, B. Dragovic, K. Fraser, S. Hand, T. Harris, A. Ho, R. Neugebauer, I. Pratt, and A. Warfield, Xen and the art of virtualization, in Proceedings of the 19th ACM Symposium on Operating Systems Principles, October, 2003. pp. 164 - 177.

[3] T. L. Borden, J. P. Hennessy, J. W. Rymarczyk, Multiple operating systems on one processor complex, IBM Systems Journal, 28(1), 1989.

[4] The Home of Checkpointing Packages, http://www.checkpointing.org

[5] P. M. Chen, B. D. Noble, When virtual is better than real, in Proceedings of the 2001 Workshop on Hot Topics in Operating Systems (HotOS-VIII), May 2001.

[6] C. Clark, K. Fraser, S. Hand, J. G. Hansen, E. Jul, C. Limpach, I. Pratt, and A. Warfield, Live Migration of Virtual Machines, in Proceedings of the 2nd Symposium on Networked Systems Design and Implementation (NSDI), May 2005.

[7] R. J. Creasy, The Origin of the VM/370 Time-Sharing System, IBM Journal of Research and Development, September 1981, pp. 483 - 490.

[8] J. Dike, A user-mode port of the Linux kernel, in Proceedings of the 4th USENIX Annual Linux Showcase and Conference, Atlanta, GA, USA, October 2000.

[9] J. Dike, The User-mode Linux Kernel Home Page, http://user-mode-linux.sourceforge.net/.

[10] R. P. Goldberg, Survey of Virtual Machine Research, IEEE Computer Magazine, vol. 7, no. 6, June 1974, pp. 34 - 45.

[11] M. R. Guthaus, J. S. Ringenberg, D. Ernst, T. M. Austin, T. Mudge, and R. B. Brown, MiBench: A free, commercially representative embedded benchmark suite, in IEEE 4th Annual Workshop on Workload Characterization, December 2001.

[12] P. H. Hargrove, J. C. Duell, Berkeley Lab Checkpoint/Restart (BLCR) for Linux Clusters, in Proceedings of SciDAC 2006, June 2006.

[13] K. Lawton et al., Bochs IA-32 Emulator Project, http://bochs.sourceforge.net

[14] Microsoft Corporation, Virtual Server 2005 R2 Technical Overview, http://download.microsoft.com/download/5/5/3/55321426cb43-4672-9123-74ca3af6911d/VS2005TechWP.doc, December 2005.

[15] A. Nagarajan, F. Mueller, C. Engelmann, and S. L. Scott, Proactive fault tolerance for HPC with Xen virtualization, in Proceedings of the 21st ACM International Conference on Supercomputing (ICS) 2007, Seattle, WA, USA, June 2007.

[16] Plex86 x86 Virtual Machine Project, http://plex86.sourceforge.net

[17] R. Potter, One-Click Distribution of Preconfigured Linux Runtime State, in WIP Session of the 3rd Virtual Machine 
Research and Technology Symposium (VM04), San Jose, CA, USA, May 2004.

[18] C. P. Sapuntzakis, R. Chandra, B. Pfaff, J. Chow, M. S. Lam, and M. Rosenblum, Optimizing the Migration of Virtual Computers, in Proceedings of the 5th Operating Systems Design and Implementation, Boston, MA, USA, December 2002, pp 377 - 390.

[19] SBUML, ScrapBook for User-Mode Linux, $\mathrm{http}: / /$ sbuml.sourceforge.net/.

[20] A. Singh, An Introduction to Virtualization, http://www.kernelthread.com/publications/virtualization.

[21] J. E. Smith, R. Nair, Virtual Machines: Versatile Platforms For Systems and Processes, Morgan Kaufmann, San Francisco, CA, USA, 2005.

[22] Sun Containers: Server Virtualization and Manageability, A Technical White Paper, http://www.sun.com/software/whitepapers/solaris10/grid_co ntainers.pdf, Sun Microsystems, September 2004.

[23] VMware ESX Server, http://www.vmware.com/products/vi/esx/, VMware, Inc.
[24] VMware VirtualCenter, http://www.vmware.com/products/vi/vc/, VMware, Inc.

[25] M. Welsh, D. Culler, Adaptive overload control for busy internet servers, in Proceedings of the 4th USENIX Conference on Internet Technologies and Systems, Seattle, WA, USA, March 2003.

[26] S. C. Woo, M. Ohara, E. Torrie, J. P. Singh, and A. Gupta, The SPLASH-2 Programs: Characterization and

Methodological Considerations, in Proceedings of the 22nd International Symposium on Computer Architecture, pp. 2436, Santa Margherita Ligure, Italy, June 1995.

[27] T. Wood, P. Shenoy, A. Venkataramani, and M. Yousif, Black-box and gray-box strategies for virtual machine migration, in Proceedings of the 4th Usenix Symposium on Networked System Design and Implementation (NSDI), Cambridge, MA, April 2007.

[28] The Xen Virtual Machine Monitor, http://www.cl.cam.ac.uk/Research/SRG/netos/xen/, University of Cambridge Computer Laboratory. 